\title{
Sp(4) gauge theories and beyond the standard model physics
}

\author{
Biagio Lucini ${ }^{1,2, *}$, Ed Bennett $^{2}$, Jack Holligan ${ }^{3,4}$, Deog Ki Hong ${ }^{5}$, Ho Hsiao ${ }^{6}$, Jong-Wan \\ Lee $^{5,7}$, C.-J. David Lin ${ }^{6,8,9}$, Michele Mesiti ${ }^{2}$, Maurizio Piai ${ }^{3}$, and Davide Vadacchino ${ }^{10}$ \\ ${ }^{1}$ Department of Mathematics, Swansea University, Fabian Way, SA1 8EN Swansea, Wales, UK \\ ${ }^{2}$ Swansea Academy of Advanced Computing, Swansea University, Fabian Way, SA1 8EN Swansea, \\ Wales, UK \\ ${ }^{3}$ Department of Physics, Swansea University, Singleton Park, SA2 8PP Swansea, Wales, UK \\ ${ }^{4}$ Department of Physics, University of Maryland, College Park, Maryland, USA, 20742 \\ ${ }^{5}$ Department of Physics, Pusan National University, Busan 46241, Korea \\ ${ }^{6}$ Institute of Physics, National Yang Ming Chiao Tung University, 1001 Ta-Hsueh Road, Hsinchu \\ 30010, Taiwan \\ ${ }^{7}$ Extreme Physics Institute, Pusan National University, Busan 46241, Korea \\ ${ }^{8}$ Center for High Energy Physics, Chung-Yuan Christian University, Chung-Li 32023, Taiwan \\ ${ }^{9}$ Centre for Theoretical and Computational Physics, National Yang Ming Chiao Tung University, 1001 \\ Ta-Hsueh Road, Hsinchu 30010, Taiwan \\ ${ }^{10}$ School of Mathematics and Hamilton Mathematics Institute, Trinity College, D02 PN40 Dublin 2, \\ Ireland
}

\begin{abstract}
We review numerical results for models with gauge group $\operatorname{Sp}(2 N)$, discussing the glueball spectrum in the large- $N$ limit, the quenched meson spectrum of $S p(4)$ with Dirac fermions in the fundamental and in the antisymmetric representation and the $S p(4)$ gauge model with two dynamical Dirac flavours. We also present preliminary results for the meson spectrum in the $S p(4)$ gauge theory with two fundamental and three antisymmetric Dirac flavours. The main motivation of our programme is to test whether this latter model is viable as a realisation of Higgs compositeness via the pseudo Nambu Goldstone mechanism and at the same time can provide partial top compositeness. In this respect, we report and briefly discuss preliminary results for the mass of the composite baryon made with two fundamental and one antisymmetric fermion (chimera baryon), whose physical properties are highly constrained if partial top compositeness is at work. Our investigation shows that a fully non-perturbative study of Higgs compositeness and partial top compositeness in $S p(4)$ is within reach with our current lattice methodology.
\end{abstract}

\section{Introduction}

$S p$ (4) gauge theory with two fundamental and three antisymmetric Dirac fermion flavours has been suggested as a possible template [1-3] for beyond the standard model strong interactions that can give rise to a composite Higgs boson through the breaking of the global fundamental flavour symmetry [4] and at the same time to a partial composite top quark state

\footnotetext{
*Speaker; e-mail: b.lucini@swansea.ac.uk
} 
that explains why the observed mass of the latter particle is at the electroweak scale [5]. Partial top compositeness results from the mixing of the standard model top quark with a chimera baryon, i.e. a baryonic state formed with two fermions in the fundamental and one fermion in the antisymmetric representation. A necessary ingredient for partial top compositeness is the generation of a large anomalous dimension for the chimera baryon. Lattice gauge theory can be used as a framework to study non-perturbatively these phenomena, in order to assess their viability beyond semi-quantitative arguments.

In this work, we shall discuss our previous and current lattice investigations of the composite Higgs mechanism and of partial top compositeness using $S p(4)$ gauge theory as a concrete realisation (for studies involving $S U(4)$, see, e.g., [6-8]), starting from special cases of the target action and approaching the latter in a crescendo of complexity. The work is organised as follows. In Sect. 2 we introduce the lattice model. Numerical results are presented in Sect. 3. Finally, we draw our conclusions and we give a brief outlook of forthcoming numerical investigations in Sect. 4.

\section{Lattice formulation and observables}

The lattice action we have used in our calculations in $\operatorname{Sp}(2 N)$ gauge theories with fermions in multiple representations $R$ can be written as

$$
S=\beta \sum_{x} \sum_{\mu<v}\left(1-\frac{1}{2 N_{c}} \mathfrak{R} \operatorname{Tr} \mathcal{P}_{\mu \nu}\right)+\sum_{j=1}^{N_{f}^{R}} \sum_{x, y} \bar{\Psi}_{j}^{R}(x) D^{R}(x, y) \Psi_{j}^{R}(y) .
$$

The first term is the Wilson plaquette action, with $\beta=2 N_{c} / g^{2}$, where $N_{c}=2 N$ is the number of colours and $g$ is the gauge coupling, $\mathcal{P}_{\mu \nu}(x)$ is the path exponential of the link variables $U_{\mu}(x) \in S p(2 N)$ around the elementary plaquette stemming from point $x$ in directions $\mu, v$ and $\mathfrak{R T r}$ indicates the real part of the trace. The second term is the fermionic part of the action. This term includes a sum over the representations $R$ and over the flavours $N_{f}^{R}$ at fixed representation. For the Dirac operator $D(x, y)$ we use the Wilson discretisation, given by

$$
D^{R}(x, y)=\left(4+a m_{0}^{\mathrm{R}}\right) \delta_{x, y}-\frac{1}{2} \sum_{\mu}\left\{\left(1-\gamma_{\mu}\right) U_{\mu}^{R}(x) \delta_{x+\hat{\mu}, y}+\left(1+\gamma_{\mu}\right) U_{\mu}^{R^{\dagger}}(y) \delta_{x-\hat{\mu}, y}\right\},
$$

where $U_{\mu}^{R}(X)$ is the gauge link variable in the representation $R, a$ is the lattice spacing and $m_{0}^{R}$ is the bare mass of the $N_{f}^{R}$ degenerate flavours in representation $R$.

The path integral of the theory can be expressed as

$$
Z=\int(\mathcal{D} U)\left(\mathcal{D} \Psi^{R}\right)\left(\mathcal{D}^{R}\right) e^{-S}
$$

where the integral is performed over all fields and the action, $S$, is given by Eq. (1). In this work, we consider two flavours of fermions in the fundamental representation $R \equiv F$ and three flavours of fermions in the antisymmetric representation $R \equiv A$.

Given an operator $O\left(x_{1}, \ldots, x_{n}\right)$, which is a function of fields at points $x_{1}, \ldots, x_{n}$, its vacuum expectation value is computed as

$$
\left\langle O\left(x_{1}, \ldots, x_{n}\right)\right\rangle=\frac{1}{Z} \int(\mathcal{D} U)\left(\mathcal{D} \Psi^{R}\right)\left(\mathcal{D}^{R}\right) O\left(x_{1}, \ldots, x_{n}\right) e^{-S},
$$

where the path integral is calculated using Monte Carlo importance sampling.

We shall consider the following observables: 


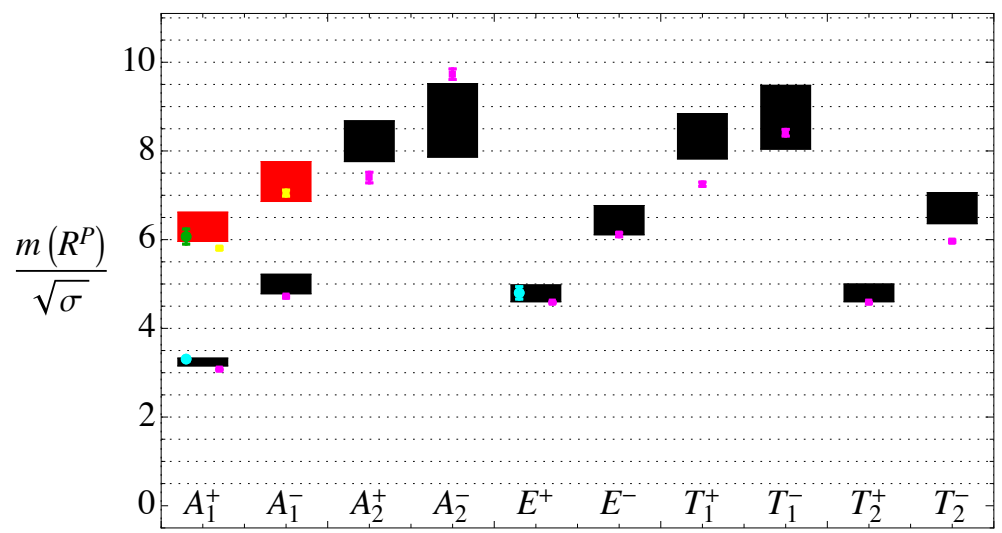

Figure 1. The $S p(2 N)$ glueball spectrum in the large- $N$ limit (boxes, with ground states in each channel depicted in black and excitations in red) compared with $S U(N)$ data extrapolated to the same limit (circles with error bars). See text for further details.

- Glueball correlators, which take the form

$$
C_{i j}\left(x_{1}, x_{2}\right)=\left\langle O_{i}\left(x_{1}\right) O_{j}^{\dagger}\left(x_{2}\right)\right\rangle-\left\langle O_{i}\left(x_{1}\right)\right\rangle\left\langle O_{j}^{\dagger}\left(x_{2}\right)\right\rangle,
$$

where $O_{i}\left(x_{1}\right)$ and $O_{j}\left(x_{2}\right)$ are combinations of Wilson loops transforming in an irreducible representation of the octahedral group, at zero momentum and with defined parity;

- Meson correlators, which are expressed as

$$
C_{\Gamma_{1} \Gamma_{2}}^{R}\left(x_{1}, x_{2}\right)=\left\langle\bar{\Psi}^{R}\left(x_{1}\right) \Gamma_{1} \Psi^{R}\left(x_{1}\right)\left(\bar{\Psi}^{R}\left(x_{2}\right) \Gamma_{2} \Psi^{R}\left(x_{2}\right)\right)^{\dagger}\right\rangle,
$$

with $\Gamma_{1}$ and $\Gamma_{2}$ combinations of Euclidean Dirac matrices, whose specific form determines the $J^{P C}$ quantum numbers of the states that saturate the propagator;

- Chimera baryon correlator, written as

$$
C_{T}^{F F A}\left(x_{1}, x_{2}\right)=\left\langle\left(T \Psi^{F}\left(x_{1}\right) \Psi^{F}\left(x_{1}\right) \Psi^{A}\left(x_{1}\right)\right)\left(T \Psi^{F}\left(x_{2}\right) \Psi^{F}\left(x_{2}\right) \Psi^{A}\left(x_{2}\right)\right)^{\dagger}\right\rangle,
$$

where $T$ is a tensor contracting flavour and colour indices, which, for the sake of conciseness, will be left implicit. More details on this construction will be provided in a forthcoming publication.

\section{Numerical Results}

Before tackling the full theory, relevant operators have been studied in notable limits of the latter. Since the properties of the model in these limits are better known, the simplified calculations have enabled us to develop the methodology and at the same time to provide a set of safe reference measurements against which to check the complete calculations. Here, we report a selection of our numerical results. For additional measurements and their discussion, we refer to the quoted original papers. 


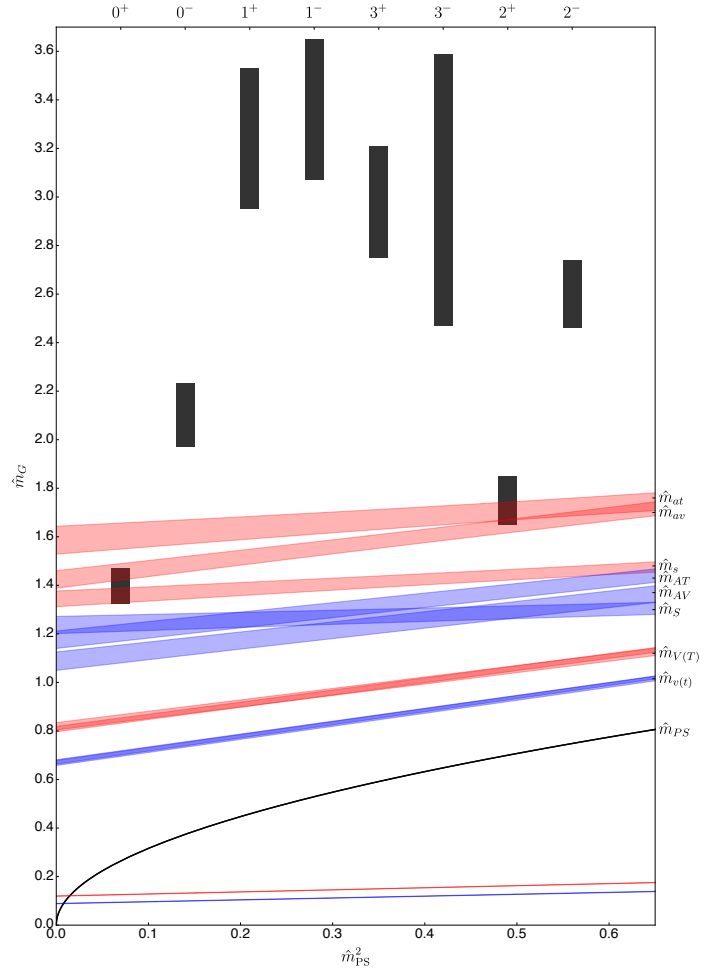

Figure 2. Quenched spectrum for $S p(4)$ gauge theory with fermions in the fundamental and in the antisymmetric representation extrapolated to the continuum limit as a function of the pseudoscalar mass squared (in black). The bands account for the statistical errors. The fundamental spectrum is drown in blue, while the antisymmetric spectrum is represented in red. Observables are labelled in the right vertical axis. Glueball masses are also reported (boxes), with quantum numbers labelled on the top horizontal axes. Note that, while there is a dependency of mesonic quantities from the pion mass, since the theory is quenched, the glueball spectrum is constant as a function of the constituent fermion mass.

The first model we have considered is the pure gauge system (first investigated in [9]). In this case, relevant observables are glueball masses and the string tension. These are extracted from correlators of the type given in Eq. (5), looking at the asymptotic behavior

$$
C_{i j}\left(x_{1}, x_{2}\right) \underset{\left|x_{1}-\bar{x}_{2}\right| \rightarrow \infty}{\simeq} C e^{-M\left|x_{1}-x_{2}\right|},
$$

where $M$ is the mass of the lowest-lying state carrying the quantum numbers of the source operators. In practice, the calculation of single correlators is very noisy. The bad signal-tonoise ratio can be overcome with a thorough variational calculation. The methodology we have used is explained in [10]. The string tension can be extracted similarly, considering correlators of Polyakov loops.

We have computed in [10] the lowest-lying glueball spectrum in units of the string tension in the continuum limit for $\operatorname{Sp}(2 N)$ models with $N=2,3,4$ and we have extrapolated the latter to $N \rightarrow \infty$. For the large- $N$ extrapolation, we have found that the leading expected $1 / N$ correction provides a good description of the data. Our results are displayed by the boxes of Fig. 1. The black boxes indicate the masses of the groundstate in each channel, with the red 


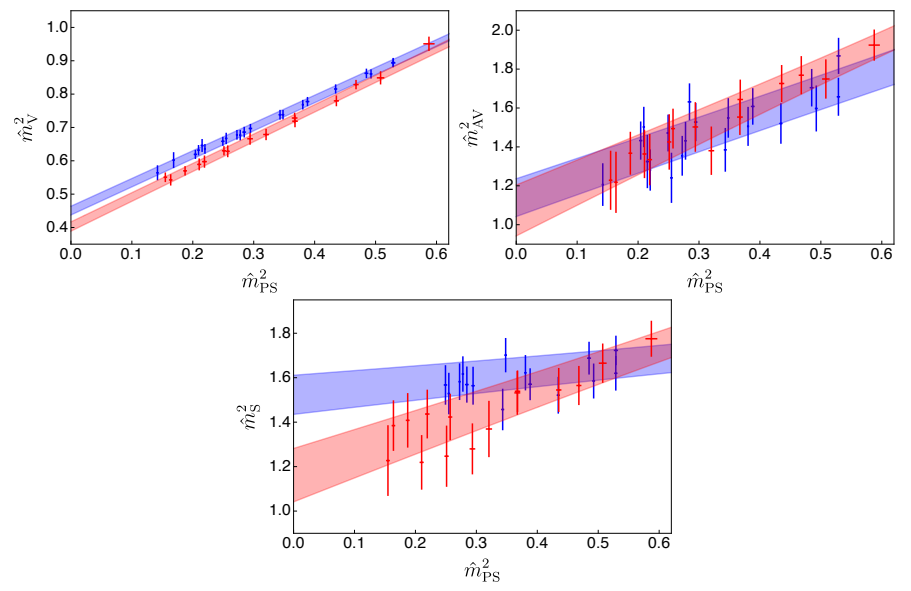

Figure 3. Squared masses of the vector (top left), the axial vector (top right) and the isotriplet scalar meson (bottom) as a function of the squared mass of the pseudoscalar for the fundamental representation in the continuum limit. We plot in red dynamical data and in blue quenched data, with the corresponding bands representing the chiral extrapolation.

boxes representing masses of the excited states (for the channels for which the latter turned out to be measurable on our samples). The quantum numbers refer to the irreducible representations of the octahedral group. The continuum $J^{P}$ quantum numbers ${ }^{1}$ can be reconstructed following the method described, e.g., in [11].

It is worth remarking that, since the large- $N$ limit of $S U(N)$ and $S p(2 N)$ coincide, for the majority of the glueball states, our work is the first extrapolation of the glueball spectrum to $N=\infty$. Indeed, the only states whose $S U(N)$ large- $N$ mass had been determined before our calculations are the groundstate and the first excitation in the spin zero channel (in our notation, the $A_{1}$ channel) and the spin two (which appears in both the $E$ and the $T_{2}$ channel) $[12,13]$ (see also [14]). We report in Fig. 1 the more constraining measurements of [13] (in cyan for the groundstates and in green for the excitation in the $A_{1}^{+}$channel), which are in full agreement with ours. After our work was published, a comprehensive study of the large- $N S U(N)$ glueball spectrum was published [15]. This enables us to perform a broader comparison of the two extrapolations. The results of [15] are also reported in Fig. 1 (in magenta for the groundstates, and in yellow for the excitations). While we notice that the authors of [15] quote significantly smaller errors, the agreement with our measurements is within the error bars (as measured on our own data) in most of the cases, with the largest discrepancies being at most around two standard deviations. This comparison provides evidence of the universality of the large- $N$ glueball spectrum across the $S U(N)$ and the $S p(2 N)$ series.

We now discuss the quenched spectrum of the $S p(4)$ gauge theory, comparing masses of glueballs and mesons, the latter studied as the mass of the constituent fermions is decreased. We consider a mesonic correlators of the form (6). Its asymptotic behaviour is given by

$$
C_{\Gamma_{1} \Gamma_{2}}^{F}\left(x_{1}, x_{2}\right) \simeq A\left(e^{-M t}+e^{-M(T-t)}\right)
$$

\footnotetext{
${ }^{1}$ We remind the reader that, since $\operatorname{Sp}(2 N)$ groups are pseudoreal, the charge conjugation quantum number of glueballs in $\operatorname{Sp}(2 N)$ is always +1 .
} 

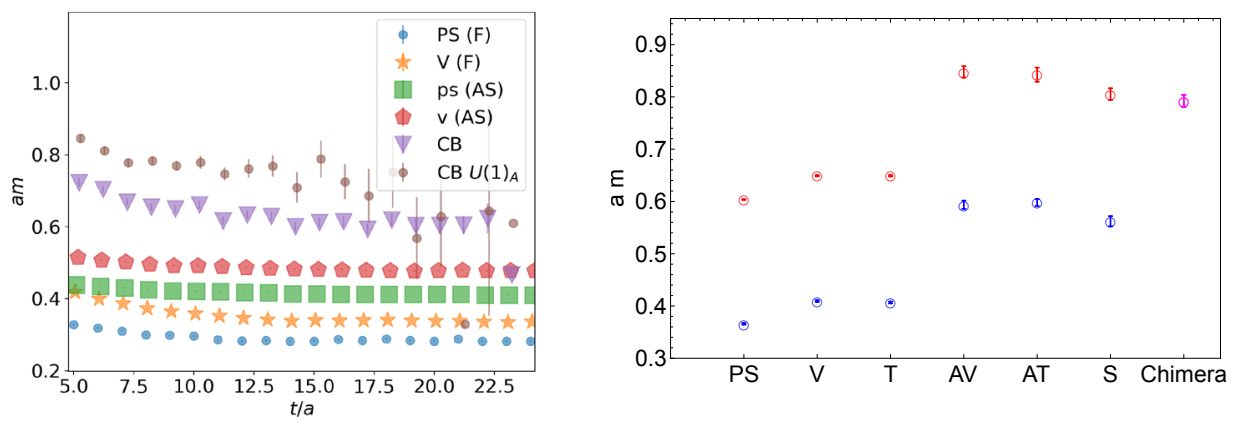

Figure 4. Left: The lowest-lying meson spectrum in $S p(4)$ with three dynamical antisymmetric fermions. Taking quenched fundamental sources in this model, we also show the pseudoscalar and the vector meson masses in the fundamental representation, the chimera baryon $(\mathrm{CB})$ and its $U(1)_{A}$ partner. The lattice parameters are discussed in the text. Right: The meson spectrum for both representations and the chimera baryon in the $S p(4)$ gauge theory with two fundamental dynamical flavours and three antisymmetric dynamical flavours, at the lattice parameters discussed in the text.

where the second term (due to waves travelling across the antiperiodic boundary of our lattice) has now been inserted explicitly, as, unlike in the glueball case, neglecting it would give a measurable systematic error. In the previous formula, $T$ is the temporal extension of the lattice. Points $x_{1}$ and $x_{2}$ have spacial coordinates summed over $\left|\vec{x}_{1}-\vec{x}_{2}\right|$ at fixed $\vec{x}_{2}$ (i.e., the correlator has zero net momentum). The temporal coordinates are respectively $\tau$ and $t+\tau$ at fixed $\tau<T$. The decay constant of the considered states can be extracted from $A$, either directly or (in the case of the pseudoscalar meson) considering two appropriate combinations of correlators. We refer to the quoted original works for the details, including renormalisation of decay constants and how the choice of the $\Gamma$ matrices identifies the $J^{P C}$ quantum numbers.

The continuum quenched meson spectrum (in both the fundamental and antisymmetric representation), originally discussed in [16], is reported in Fig. 2, together with a selection of glueball states taken from [17]. The scale is set using the gradient flow derived quantity $w_{0}$, as described in [18]. The meson spectrum in both representations is plotted as a function of the pseudoscalar mass in that representation. Glueball states are also reported for the same model. The data show some clear indications: (1) in both representations, the general features of the quenched spectrum are qualitatively similar to those of QCD; (2) while the meson spectrum has the same behaviour in both representations, at fixed pseudoscalar mass antisymmetric states are around 10-20\% heavier than the corresponding fundamental states, with the percent difference increasing towards the light fermion regime; (3) glueballs are generally significantly heavier than mesons, but the lightest glueball states have masses that are comparable with masses of states in the heaviest mesonic channels.

We have then studied the theory with fermionic matter, comparing the two-flavour dynamical model [19] to the quenched case [18] for fundamental representation fermions in the continuum limit. The masses of the vector, axial vector and isotriplet scalar meson are plotted in Fig. 3. With the exception of the latter, typically unquenching effects are very small, and hardly visible in the range of the obtained pion masses.

In the subsequent stage of our investigation programme of $S p(4)$ gauge models, we have studied the system in a partially quenched setup, considering three dynamical antisymmet- 
ric fermions and two static fundamental flavours. This gives us an opportunity to perform a first study of the chimera baryon in a model that is less complicated to simulate than the target mixed-representation system. Figure 4 (left) displays our preliminary results for the two lowest-lying mesonic states (the pseudoscalar and the scalar) in both representations, together with the chimera baryon and its parity partner. Results have been obtained on a $48 \times 24^{3}$ lattice at gauge coupling $\beta=6.65$, antisymmetric fermion bare mass $a m_{0}^{A}=-1.07$ and quenched fundamental mass $a m_{0}^{F}=-0.734$. Statistical noise has been reduced using Wuppertal smearing [20]. The data show that in our theory and for the used lattice parameters, chimera baryons are heavier than the antisymmetric vector meson. Beyond the (not unimportant) numerical details, likely to be dependent on our particular setup, the main conclusion of our investigation is that the technology we have developed enables us to determine masses of chimera baryons. Note in particular the visible splitting between the chimera baryon and its $U(1)_{A}$ partner.

Finally, we are now performing calculations in $S p(4)$ gauge theory with two dynamical fundamental fermions and three dynamical antisymmetric fermions. A preliminary plot the meson spectrum and of the chimera baryon is reported in Fig. 4, right. The lattice parameters are $\beta=6.5, a m_{0}^{A}=-1.01$ and $a m_{0}^{F}=-0.71$, and the lattice size is $48 \times 24^{3}$. The results show that for our choice of parameters the chimera baryon has a mass comparable to that of the axial vector and of the isotriplet scalar mesons in the antisymmetric representation.

\section{Conclusions and outlook}

Following the programme we started in [18], we have performed a set of extensive simulations aimed to assess the viability of $S p(4)$ gauge theory with two fundamental and three antisymmetric fermions as a realisation of the composite Higgs and of the partial top compositeness mechanism. So far we have developed the needed technology by studying the glueball spectrum [17], the quenched meson spectrum [16] and the dynamical theories respectively with two fundamental [19] and three antisymmetric fermion flavours. A selection of the results is reported in this work. A more systematic calculation in the target phenomenological model, which includes dynamical fermions in both representations, is now within reach. We have presented some preliminary results for the latter model. A more detailed account of its features will be reported in a forthcoming publication. Another interesting direction, pursued by the authors of [21], is to explore an $S p$ (4) gauge models with two non-degenerate fundamental fermions in the context of SIMP dark matter.

Acknowledgements - The work of E. B. has bee $\mathrm{n}$ funded by the Supercomputing Wales project, which is part-funded by the European Regional Development Fund (ERDF) via Welsh Government. J. H. is supported by the STFC Consolidated Grant No. ST/P00055X/1, by the College of Science, Swansea University, and by the Grant No. STFC-DTG ST/R505158/1. The work of D. K. H. was supported by Basic Science Research Program through the National Research Foundation of Korea (NRF) funded by the Ministry of Education (NRF-2017R1D1A1B06033701). The work of J. W. L. is supported in part by the National Research Foundation of Korea funded by the Ministry of Science and ICT (NRF-2018R1C1B3001379) and in part by Korea Research Fellowship program funded by the Ministry of Science, ICT and Future Planning through the National Research Foundation of Korea (2016H1D3A1909283). The work of C. J. D. L. is supported by the Taiwanese MoST Grant No. 105-2628-M-009-003- MY4. The work of B. L. and M. P. has been supported in part by the STFC Consolidated Grant No. ST/T000813/1. B. L. and M. P. received funding from the European Research Council (ERC) under the European Union's Horizon 2020 research and innovation program under Grant Agreement No. 813942. The work of B. L. is further supported in part by the Royal Society Wolfson Research Merit Award No. WM170010 and by the Leverhulme Trust Research Fellowship 
No. RF-2020-4619. The work of D. V. is supported in part by the INFN HPCHTC project and in part by the Simons Foundation under the program "Targeted Grants to Institutes" awarded to the Hamilton Mathematics Institute. Numerical simulations have been performed on the Swansea SUNBIRD cluster (part of the Supercomputing Wales project) and AccelerateAI A100 GPU system, on the local HPC clusters in Pusan National University (PNU) and in National Chiao-Tung University (NCTU), and on the Cambridge Service for Data Driven Discovery (CSD3). The Supercomputing Wales project and AccelerateAI are part funded by the European Regional Development Fund (ERDF) via Welsh Government. CSD3 is operated in part by the University of Cambridge Research Computing on behalf of the STFC DiRAC HPC Facility (www.dirac.ac.uk). The DiRAC component of CSD3 was funded by BEIS capital funding via STFC capital Grants No. ST/P002307/1 and No. ST/R002452/1 and STFC operations Grant No. ST/R00689X/1. DiRAC is part of the National e-Infrastructure.

\section{References}

[1] J. Barnard, T. Gherghetta, T.S. Ray, JHEP 02, 002 (2014), 1311.6562

[2] G. Ferretti, D. Karateev, JHEP 03, 077 (2014), 1312 . 5330

[3] G. Cacciapaglia, G. Ferretti, T. Flacke, H. Serôdio, Front. in Phys. 7, 22 (2019), 1902.06890

[4] D.B. Kaplan, H. Georgi, Phys. Lett. B 136, 183 (1984)

[5] D.B. Kaplan, Nucl. Phys. B 365, 259 (1991)

[6] V. Ayyar, T. DeGrand, M. Golterman, D.C. Hackett, W.I. Jay, E.T. Neil, Y. Shamir, B. Svetitsky, Phys. Rev. D 97, 074505 (2018), 1710.00806

[7] V. Ayyar, T. Degrand, D.C. Hackett, W.I. Jay, E.T. Neil, Y. Shamir, B. Svetitsky, Phys. Rev. D 97, 114505 (2018), 1801.05809

[8] G. Cossu, L. Del Debbio, M. Panero, D. Preti, Eur. Phys. J. C 79, 638 (2019), 1904.08885

[9] K. Holland, M. Pepe, U.J. Wiese, Nucl. Phys. B 694, 35 (2004), hep-lat/0312022

[10] E. Bennett, J. Holligan, D.K. Hong, J.W. Lee, C.J.D. Lin, B. Lucini, M. Piai, D. Vadacchino, Phys. Rev. D 102, 011501 (2020), 2004.11063

[11] B. Lucini, A. Rago, E. Rinaldi, JHEP 08, 119 (2010), 1007 . 3879

[12] B. Lucini, M. Teper, JHEP 06, 050 (2001), hep-lat/0103027

[13] B. Lucini, M. Teper, U. Wenger, JHEP 06, 012 (2004), hep-lat/0404008

[14] B. Lucini, M. Panero, Phys. Rept. 526, 93 (2013), 1210. 4997

[15] A. Athenodorou, M. Teper (2021), 2106.00364

[16] E. Bennett, D.K. Hong, J.W. Lee, C.J.D. Lin, B. Lucini, M. Mesiti, M. Piai, J. Rantaharju, D. Vadacchino, Phys. Rev. D 101, 074516 (2020), 1912.06505

[17] E. Bennett, J. Holligan, D.K. Hong, J.W. Lee, C.J.D. Lin, B. Lucini, M. Piai, D. Vadacchino, Phys. Rev. D 103, 054509 (2021), 2010. 15781

[18] E. Bennett, D.K. Hong, J.W. Lee, C.J.D. Lin, B. Lucini, M. Piai, D. Vadacchino, JHEP 03, 185 (2018), 1712.04220

[19] E. Bennett, D.K. Hong, J.W. Lee, C.J.D. Lin, B. Lucini, M. Piai, D. Vadacchino, JHEP 12, 053 (2019), 1909. 12662

[20] S. Gusken, Nucl. Phys. B Proc. Suppl. 17, 361 (1990)

[21] A. Maas, F. Zierler, PoS(LATTICE2021) to appear (2021), 2109. 14377 